\title{
Behavioural correlates of selection for oviposition by Drosophila melanogaster females in a patchy environment
}

\author{
GLADYS RUIZ-DUBREUIL†, BARRIE BURNET* $\ddagger$ \& KEVIN CONNOLLY§ \\ tInstituto de Ecologia y Evolucion, Universidad Austral, Valdivia, Chile; $\ddagger$ Department of Molecular Biology and \\ Biotechnology and \$Department of Psychology, University of Sheffield, Sheffield S10 2UH, U.K.
}

\begin{abstract}
The behaviour of females from lines selected for high $(\mathrm{H})$ and low $(\mathrm{L})$ aggregated oviposition was compared in an environment consisting of discrete patches of resource available for larval development. Oviposition behaviour was influenced by the conformation and by the texture of the substrate, but this does not account for the selective differences in levels of aggregation which are under genetic control. The distribution of males of both selected populations tends to be overdispersed across resource patches. This observation is consistent with male territorial behaviour. The dispersal patterns of females of the two selected populations differ significantly. $\mathrm{H}$ females show a contagious distribution whereas the distribution for $L$ females is more nearly random. Differences in adult female dispersion are likely to be a significant factor contributing to aggregated oviposition. The level of aggregated oviposition affects the pattern of progeny survival when the unit of resource in each patch is small.
\end{abstract}

Keywords: aggregated oviposition, behaviour, Drosophila melanogaster, patchy environment, selection response, spatial dispersion.

\section{Introduction}

A phenomenon which biologists are concerned to understand is the occurrence in a habitat of stable associations of different species with the same or overlapping resource requirements. The pre-adult stages of many Drosophila species occupy environments in which resources are both transient and patchy in their distribution. Explanations for the continued coexistence of two or more competing species in these circumstances are usually sought in terms of resource partitioning, or of refugia (Shorrocks, 1990), in particular 'probability refugia'. Rosewell et al. (1990) and Shorrocks et al. (1990) provide a definitive account. According to theory, two species exploiting such resource patches might never exclude one another entirely, despite competition between them, provided the pre-adult stages of both species have aggregated and independent distributions.

Females of some species of Drosophila have a tendency to lay their eggs at sites where eggs have

*Correspondence: B. Burnet, Department of Molecular Biology and Biotechnology, PO Box 594, Firth Court, Western Bank, Sheffield S10 2UH, U.K. already been laid, leading to aggregated or gregarious oviposition (Kambysellis et al., 1980; Brncic, 1987). Different environmental factors are known to affect the choice of oviposition sites by females. These include chemical cues (Gelfand \& McDonald, 1980; Jaenike, 1982), the colour (Volpe et al., 1967; del Solar \& Ruiz, 1979) and surface texture of the substrate (David, 1970; Takamura \& Fuyama, 1980), temperature (Fogelman, 1979), light intensity (Wogaman \& Seiger, 1983), as well as the presence of eggs or other preadult stages (del Solar \& Palomino, 1966; del Solar, 1968). Spatial heterogeneity of environmental cues could therefore give rise to aggregated distributions of eggs and other pre-adult stages. The distributions might be independent if species respond to different environmental cues, or respond to the same cue in different ways. A genetic basis for choice of substrate texture for oviposition has already been detected in Drosophila melanogaster by Takamura \& Fuyama (1980). del Solar (1968) also found a genetic basis for aggregated oviposition by $D$. pseudoobscura.

Atkinson (1983) argued that gregarious oviposition as described by del Solar (1968) is a response to differences in surface texture of the sites available for egg laying. On an otherwise plain surface eggs, or other 
pre-adult stages already present, could alter the surface topography so as to change texture. Aggregated oviposition arising in this way would, he argued, be trivial in any natural breeding site.

Ruiz \& del Solar (1986, 1991) observed a response to bidirectional selection for aggregated oviposition leading to populations of $D$. melanogaster with high, or low, indices of dispersion in a laboratory environment consisting of identical and discrete oviposition sites. These populations represent the products of one of the longest sustained periods of selection for any behavioural character reported in Drosophila. Prima facie, the causal factors leading to a contagious distribution of eggs over multiple identical sites might operate at various levels. Gregarious oviposition could arise if an egg already laid alters the probability that further eggs are laid at the same site. On the other hand, the pattern of egg deposition might be a function of the locomotor activity of flies, and hence the rate at which sites are visited. Moreover, reactivity or interactions between adult females over these sites could also affect the pattern of oviposition if females are either repelled or attracted to one another at the egg-laying sites. An understanding of the bases of genetic differences in aggregated oviposition behaviour within a cosmopolitan domesticated species, such as $D$. melanogaster, is obviously important in relation to inferences about the stability of the multispecies associations in which it occurs in the wild.

\section{Materials and methods}

Two populations of Drosophila melanogaster, the products of continuous mass selection for oviposition behaviour for 260 consecutive generations, $\mathrm{H}$ selected for high aggregation and $\mathrm{L}$ selected for low aggregation, were used.

Oviposition on an artificial substrate was measured using $30 \times 20 \times 13 \mathrm{~cm}$ population cages constructed from dark brown Tufnel plastic with friction grip insertion ports in the base for food pots. Each cage was fitted with a clear Perspex lid, and the end wall with a $10 \mathrm{~cm}$ diameter brass ventilation port fitted with a double-layered fine mesh brass gauze. Food pots were $3 \times 2 \mathrm{~cm}$ diameter plastic boiling tube caps filled with sterile killed-yeast-glucose-agar medium inserted into 20 ports equally spaced on a $4 \times 5$ grid. Centre to centre the pots were $5 \mathrm{~cm}$ apart. After the period allowed for oviposition by 20 females the pots were isolated in individual $10 \times 2.2 \mathrm{~cm}$ diameter glass vials and adult progeny counted.

Grape assemblies consisted of $13 \times 30 \mathrm{~cm}$ diameter clear polythene cake containers inverted on their lids. A $24 \mathrm{~cm}$ diameter Whatman filter paper disc marked with 25 equally spaced points ( $4 \mathrm{~cm}$ apart) was placed concentrically on the lid, and a $2 \mathrm{~cm}$ plastic universal container cap inverted at each numbered position to receive a freshly cut half grape placed cut side up. The cut surfaces of the grapes were seeded with a very dilute suspension of live baker's yeast. One half of each bisected grape was assigned to the $\mathrm{H}$ and the other to the $\mathrm{L}$ strain. Twenty-five flies were transferred without anaesthesia to a dry $7 \times 2.5 \mathrm{~cm}$ diameter glass vial closed by a foam-rubber bung and fixed down with Blu-Tack at the centre of the assembly. After a 30-min settling-down period the foam bung was gently raised by pulling an attached thread communicating through the top of the assembly. The first observation on the distribution of the flies in the assembly was made 60 min after raising the bung. Following egg counts each half grape was placed on a wad of moistened Whatman filter paper discs in a glass vial.

All experiments were conducted at $25^{\circ} \mathrm{C}$ under constant conditions of illumination.

\section{Results}

\section{Oviposition on an artificial substrate}

Initial characterization of the selected strains was made using killed-yeast-glucose-agar medium in pots in a population cage. This medium presents a uniform white and glass-like surface for oviposition. Two conditions of surface topography were used. In the first, CONCAVE, the medium formed a flat surface about $1 \mathrm{~mm}$ below the top of the vertical cylindrical walls of the pot. In the second, Convex, the medium was poured until it overtopped the walls of the pot and, held by surface tension, set to form a smooth dome. Oviposition rates of females selected for high ' $\mathrm{H}$ ' and low ' $\mathrm{L}$ ' levels of aggregation are shown in Table 1.

The attractiveness of the substrate available for egg laying is clearly influenced by the surface conformation. Mean oviposition rate is several-fold higher on the convex surface, the difference between conditions being statistically significant for both strains. The pattern of spatial dispersion for the eggs of $\mathrm{H}$ and $\mathrm{L}$ is aggregated in both cases, but the intensity of aggregation is very much greater for the eggs laid by $\mathrm{H}$ females. Conformation of the substrate appears to have little influence on the spatial dispersion patterns for eggs of the two strains.

The yeast-glucose-agar medium has a high nutritional value and supports a high survival rate in the $\mathrm{H}$ strain (95-98 per cent), although survival is somewhat lower (79-84 per cent) in the $\mathrm{L}$ strain. As expected at these relatively high survival rates, the pattern of dispersion of progenies closely correlates with the distribution of the eggs (Table 1). 
Table 1 Oviposition on pots containing yeast-glucose-agar medium. Twenty fertilized 3-4-day-old females were placed in a cage containing 20 pots with medium presenting a 'concave' surface, or 20 pots with medium presenting a 'convex' surface. Oviposition was measured by the mean \pm SE number of eggs per pot laid over a 24-h period. The pattern of dispersion of the eggs is indicated by the ratio of variance/mean. The mean \pm SE number of adult progeny emerging from each pot is also shown. $P$ is the probability that a difference between the number of eggs in, or adults emerging from, pots with concave versus convex surfaces is due to chance (Kruskal-Wallis test)

\begin{tabular}{lcccccc}
\hline & Eggs & Var./mean & $P$ & Adults & Var./mean & $P$ \\
\hline Strain $H$ & & & & & & \\
Concave & $9.5 \pm 4.5$ & 42.5 & & $9.3 \pm 4.5$ & 42.9 & \\
$\quad$ Convex & $38.2 \pm 11.8$ & 72.7 & & $36.4 \pm 11.1$ & 67.4 & \\
$\quad \begin{array}{l}\text { Strain } L \\
\text { Concave }\end{array}$ & $7.6 \pm 1.6$ & 6.7 & & $6.0 \pm 1.5$ & 7.1 & \\
Convex & $21.9 \pm 3.5$ & 11.0 & & $18.3 \pm 2.9$ & 9.1 & \\
\hline
\end{tabular}

\section{Activity and reactivity}

The possibility that flies of the $\mathrm{H}$ and $\mathrm{L}$ aggregation strains may differ in locomotor activity and in their response to the presence of other individuals was examined by confining fertilized females in groups of five in $5 \times 1 \mathrm{~cm}$ food vials. Connolly (1968) has shown that the incidence of preening increases in the presence of other individuals. Szebenyi (1969) gives a detailed description of the movements of which preening is composed. The incidences of locomotion and preening were recorded at 30 -min intervals over an 8 -h period. There were no significant differences in preening activity over time or between genotypes. Consequently there is no evidence for differences in the levels of social facilitation of preening between the $\mathrm{H}$ and $\mathrm{L}$ strains. Locomotor activity differed significantly between times of day $(P<0.01)$ and between genotypes $(P<0.01)$. The $\mathrm{L}$ strain females tend to be more active in the morning and the $\mathrm{H}$ strain females to be more active in the late afternoon.

The locomotor activity of individual fertilized females was measured in an open arena following an instantaneous mechanical disturbance at time zero (Fig. 1a). The mean locomotor activity during the ensuing 1-min intervals is a compound metric composed of two independent variables: amount and speed of movement (Burnet et al., 1988). Two-factor repeated measures ANOVA indicated significant change in locomotion over time $(P<0.01)$ in both strains. Figure $1 \mathrm{~b}$ shows that the incidence of preening changes significantly with time $(P<0.01)$ in both strains.
The initially high levels of locomotion and preening in the first minutes are responses induced in each individual by the mechanical stimulus. The rate at which this attenuates over time affords a measure of the reactivity. The levels of locomotion and preening by $\mathrm{L}$ females tend to be greater than in $\mathrm{H}$ females. The difference is only marginally significant for locomotion $(P \simeq 0.05)$, but is highly significant for preening $(P<0.01)$. Absence of statistically significant interaction between genotype and time, either for locomotion or preening, means that the $\mathrm{H}$ and $\mathrm{L}$ strains are about equally reactive to disturbance.

\section{Behaviour on a natural substrate}

Distribution of adult flies. The pattern of dispersion of flies over a natural substrate which is subdivided into discrete patches was observed using cut half grapes equally spaced over a circular area. Counts were made at hourly intervals of the number of flies on individual grapes. Each assembly contained 25 male flies of a single strain, and two replicate assemblies were used for each strain. Figure 2 shows the dispersal pattern of 3-4-day-old male flies. The horizontal line in the figure parallel to the abscissa indicates the expected value of the ratio variance $/$ mean $(\mathrm{v} / \mathrm{m})$ for flies distributed at random. The behaviour of males of the $\mathrm{H}$ and $\mathrm{L}$ strains is remarkably concordant. During the first 3-4 h the distribution tends to be aggregated $(\mathrm{v} / \mathrm{m}<1)$ whereas subsequently the trend shifts below the random expectation towards overdispersion $(\mathrm{v} / \mathrm{m}>1)$ in which males are more evenly distributed over the grapes. 
(a)

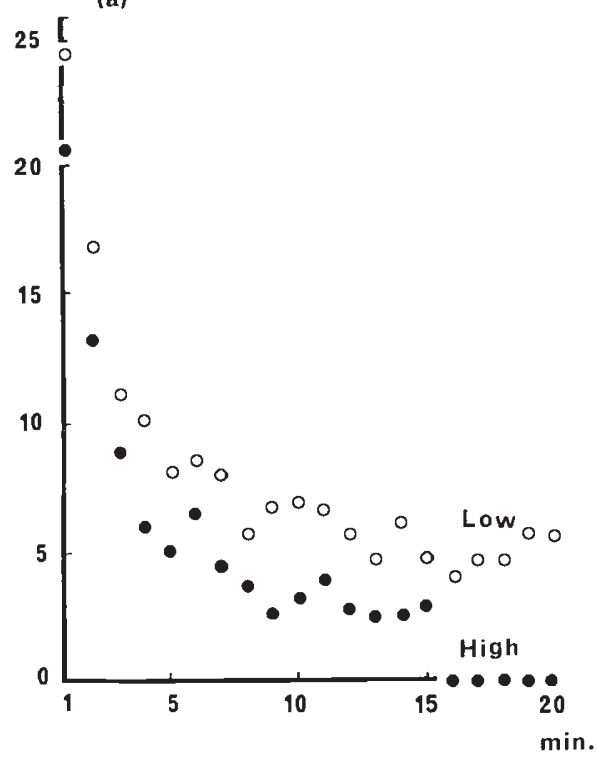

(b)

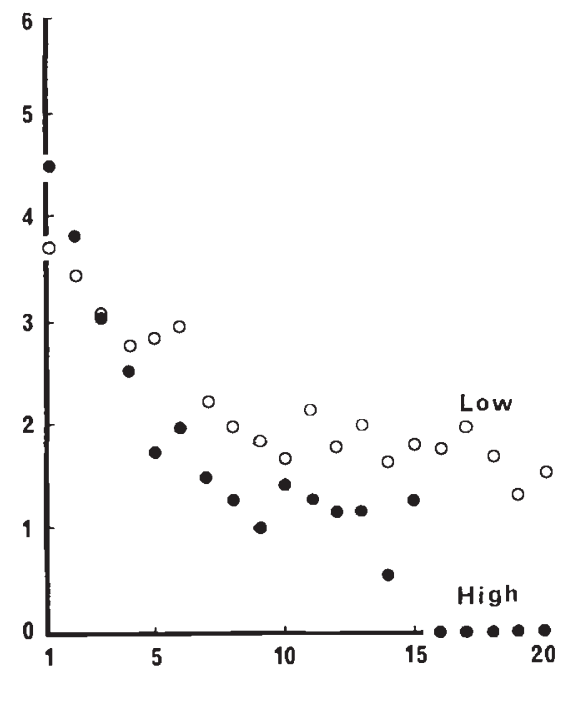

Fig. 1 (a) Locomotor activity, and (b) preening activity, of 3-day-old fertilized females observed singly in a $10 \times 10 \mathrm{~cm}$ chamber. A mechanical stimulus was given at time zero. Locomotion is expressed as the mean number of $1 \mathrm{~cm}$ squares entered per minute, and based on 30 flies of each strain. Preening is expressed as the mean number of events per minute. Solid circles denote the $\mathrm{H}$, and open circles the L, strains.

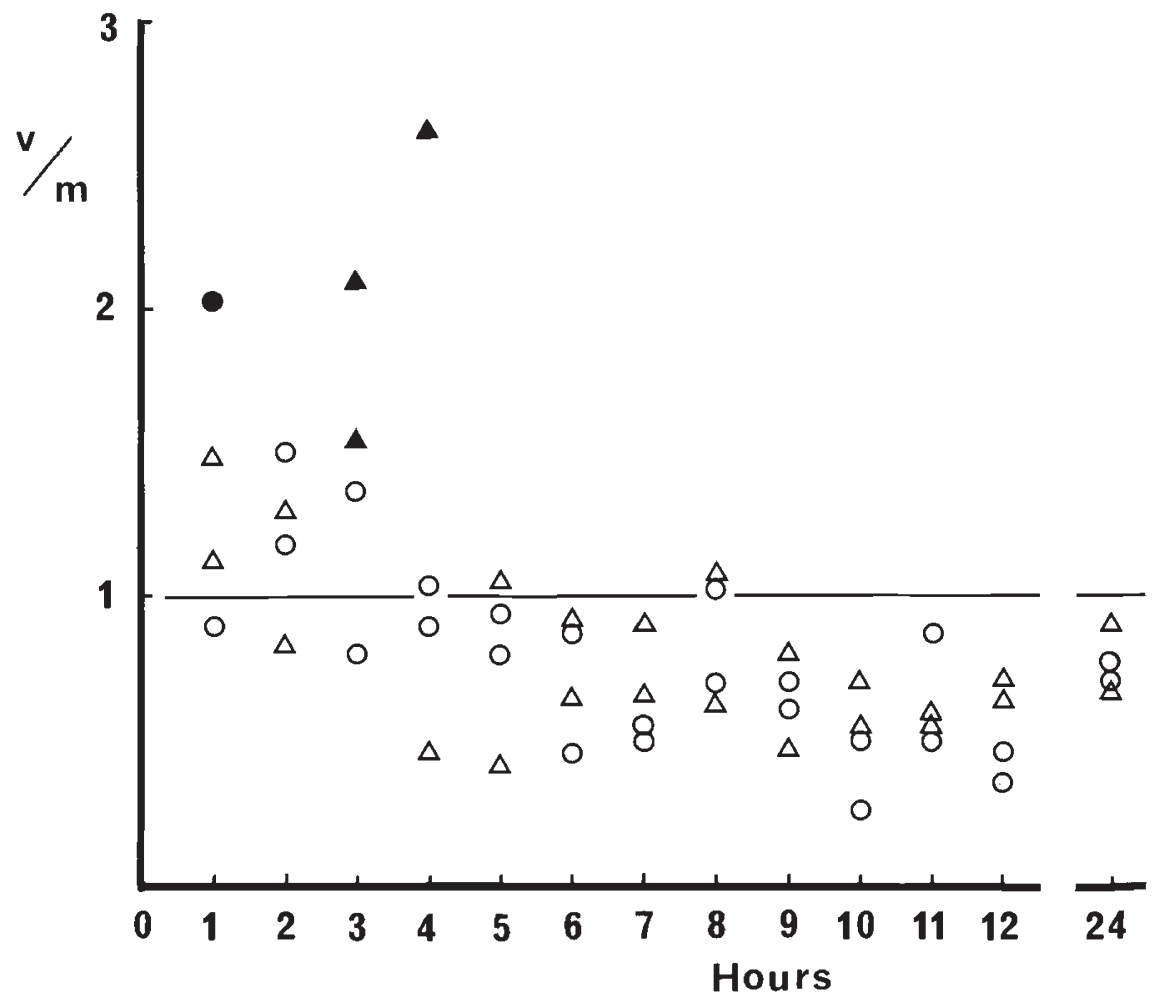

Fig. 2 Dispersal patterns in replicate grape assemblies each containing 25 male flies observed at intervals of $1 \mathrm{~h}$. The ordinate shows the index of dispersion (variance/mean). Circles denote $\mathrm{H}$ strain, triangles denote $\mathbf{L}$ strain. Black symbols identify values of the index which significantly exceed unity (random expectation) at $P<0.05$, and therefore indicate aggregation.
Figure 3 shows the distribution of 25 3-4-day-old fertilized females. Each assembly contained flies from a single strain. The dispersion patterns of $\mathrm{H}$ and $\mathrm{L}$ females contrast with each other and with those of males. The $\mathrm{H}$ strain females showed a strong tendency towards aggregation throughout the period of observa- tion, whereas L strain females tended to be distributed at random with occasional evidence of aggregation.

Oviposition. The distribution of eggs laid on grapes during $24 \mathrm{~h}$ is shown in Table 2 . The higher mean rates of oviposition relative to those in Table 1 show that 
grapes provide a more attractive substrate for egg laying than the artificial medium. A difference in mean oviposition rate between the $\mathrm{H}$ and $\mathrm{L}$ females is more clearly evident in the grape assemblies, and the inten-

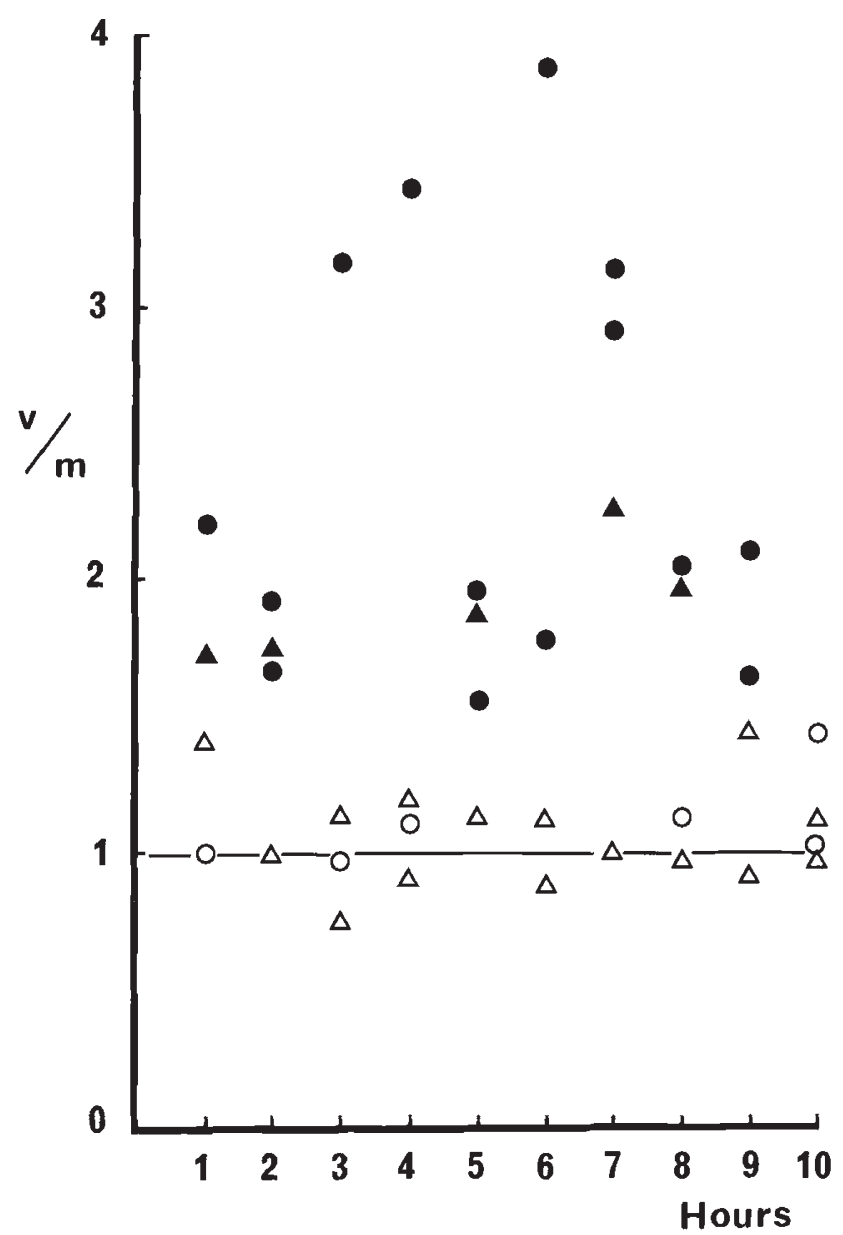

Fig. 3 Distribution of females on grapes. Replicate assemblies each contained 25 fertilized females. Refer to Fig. 2 for key. sity of aggregation is again several-fold higher for $\mathrm{H}$ compared with $\mathrm{L}$ females.

Observations on the distribution of females on grapes were made over a period of $10 \mathrm{~h}$. The correlations between number of flies observed and number of eggs laid, on individually numbered grapes, are significant (Table 2). The correlations are higher for $\mathrm{H}$ than for $\mathrm{L}$ females.

The seed effect. The texture of the surface of a bisected grape is changed by the presence of seed. The conditions with and without seed are defined exclusively in terms of whether seed was exposed on the cut surface or not. The halves of the bisected grapes were assigned to assemblies such that presence of seed was orthogonal to genotype. The results of partitioning the numbers of eggs laid on grapes with and without seed are summarized in Table 3.

Females of both strains laid more eggs on grapes with seed. For the $L$ females the difference in oviposition rate is less than the threshold for statistical significance $(P>0.05)$, whereas females of the $\mathrm{H}$ strain showed a nearly three-fold higher oviposition rate on seeded grapes $(P<0.001)$. The indices of dispersion $(\mathrm{v} / \mathrm{m})$ for the two strains indicate that the pattern of distribution of eggs is unaffected by the presence or absence of seed. The relative difference in the intensity of aggregation of eggs laid by $\mathrm{H}$ and $\mathrm{L}$ females remains the same under each condition.

\section{Survival pattern}

The mean number of adults emerged from grapes is shown in Table 2. The survival rates for both strains on grapes are lower than on the artificial medium $(\mathrm{H} 41$, 52 per cent; L 65,88 per cent), so that grapes represent suboptimal resource patches. Although the rate of oviposition on grapes is higher for the $\mathrm{H}$ strain its sur-

Table 2 Correlation between the number of flies observed on grapes in 10 inspections at hourly intervals and number of eggs laid on each grape over a 24-h period. The table shows the mean \pm SE of flies per grape, eggs per grape and progeny per grape, respectively. The patterns of dispersal of eggs and progeny are indicated by the ratio of variance $/$ mean

\begin{tabular}{lcccccc}
\hline & Flies & Eggs & Var./mean & Correlation & Progeny & Var./mean \\
\hline Replicate 1 & & & & & & \\
H & $6.92 \pm 1.88$ & $43.5 \pm 10.5$ & 62.9 & $0.87^{* *}$ & $18.0 \pm 2.66$ & 9.37 \\
L & $3.72 \pm 0.65$ & $21.6 \pm 2.93$ & 9.95 & $0.56^{* *}$ & $19.0 \pm 2.75$ & 9.92 \\
Replicate 2 & & & & & \\
H & $6.04 \pm 1.20$ & $45.2 \pm 9.28$ & 47.6 & $0.88^{* *}$ & $23.2 \pm 3.29$ & 11.7 \\
L & $4.72 \pm 0.88$ & $28.0 \pm 2.52$ & 5.71 & $0.59^{* *}$ & $18.1 \pm 1.92$ & 5.12 \\
\hline
\end{tabular}

**Denotes values of the correlation coefficient differing significantly from zero at the 1 per cent level of probability. 
Table 3 Effect of differences in surface texture, caused by the presence or absence of seeds on the cut surface of grapes, on oviposition behaviour by the $\mathrm{H}$ and $\mathrm{L}$ strains. $P$ is the probability that a difference in number of eggs laid on grapes with seed versus those without seed is due to chance (Kruskal-Wallis test)

\begin{tabular}{|c|c|c|c|c|c|c|}
\hline & Eggs & $\begin{array}{c}\text { Strain H } \\
\text { Var./mean }\end{array}$ & $P$ & Eggs & $\begin{array}{c}\text { Strain L } \\
\text { Var./mean }\end{array}$ & $P$ \\
\hline With seed & $65.2 \pm 11.1$ & 47.4 & \multirow{2}{*}{$<0.001$} & $24.1 \pm 2.61$ & 9.30 & \multirow{2}{*}{0.15} \\
\hline Without seed & $22.0 \pm 6.03$ & 41.3 & & $18.7 \pm 2.29$ & 4.68 & \\
\hline
\end{tabular}

vival rate is lower than in the $\mathrm{L}$ strain. Oviposition by $\mathrm{H}$ strain females more frequently overloads the available unit of resource at the higher egg densities causing severe reduction in survival. The numbers of eggs laid per grape by $L$ females fall within a more limited and lower range. Even at low density the larval survival in both strains is quite low on some grapes, reflecting differences in the growth of yeast in the pulp of the grapes, or differences in the rate of desiccation of the pulp.

\section{Discussion}

In addition to the texture, the conformation or topography of a substrate has an important influence on its attractiveness as an oviposition site to D. melanogaster. The effects of these properties on females of the two selected populations manifest themselves only in terms of differences in the numbers of eggs laid, whereas differences in their pattern of dispersion are largely unaffected. Heterogeneity of conformation and texture could affect the likelihood of aggregated oviposition in the natural state but, on the evidence presented here, fails to account for the phenotypic differences between the selected lines.

According to Atkinson (1983) the presence of an egg or eggs alters the surface texture of the substrate in a manner which increases the probability that further oviposition will occur at the same place. Heterogeneity of substrate texture is thus generated by the act of oviposition itself, and aggregated oviposition behaviour by single isolated females, such as observed by del Solar \& Ruiz (1992), could be interpreted as a response by females to the presence of their own eggs.

If the stimulus for contagious oviposition is simply a difference in texture caused by the presence of eggs it would be unlikely to lead to independently aggregated distributions for different genotypes or species unless they are genetically programmed to respond to this cue in different ways. Such a possibility is compatible with the findings of the present investigation. Divergent directional selection for aggregated oviposition behaviour may indeed have given rise to populations which have diverged genetically in the extent to which females are stimulated to oviposit in sites already occupied by their own eggs, or eggs laid by other females, but an empirical test of this has yet to be made.

The difference in the pattern of spatial dispersion of males and females observed in the present study agrees with results obtained using other conditions by Navarro \& del Solar (1975). Social interactions between males, involving aggressive behaviour and territoriality, are described by Hoffmann (1987), and would be expected to lead to males being overdispersed. Female flies, on the other hand, show a significant tendency towards an aggregated or contagious distribution over discrete patches of resource. This is consistent with the possibility of some form of social aggregation. The responses to selection for different levels of aggregated oviposition are associated with differences between the $\mathrm{H}$ and $\mathrm{L}$ lines in the intensity of aggregation of the females themselves. It is noteworthy that the effect is particular to the females, because no correlated changes in dispersal pattern of males appear to have occurred in the selected lines. Questions for closer scrutiny are whether females tend to aggregate because they are attracted to other females (or aggregate because they are influenced by some other environmental cue) and whether contagion in the distribution of females is causally related to aggregated oviposition.

D. melanogaster females have a diurnal rhythm in oviposition which increases towards onset of darkness to a peak in the early scotophase, then declines towards the onset of dawn (David et al., 1983). Any tendency for females to be attracted to oviposition sites already occupied by other females by responding to visual cues could be expected to be effective during the hours of daylight, but to be inoperative during the peak period of oviposition, because this occurs during the hours of darkness. However, females may also respond to nonvisual (e.g. pheromonal) indications of the presence of 
other females. In this connection it would be interesting to know what effect mutant genes disrupting normal vision or olfaction have on the spatial distribution and aggregated oviposition behaviour of the selected lines.

Spontaneous locomotor activity could cause differences in the levels of aggregated oviposition, if more active females spend less time on a larger number of oviposition sites. Females of the $\mathrm{L}$ strain tend to be more active in the first half of the day, but females of the $\mathrm{H}$ line are more active later in the day. If increased locomotor activity of the $\mathrm{H}$ line is sustained during the peak period of oviposition in scotophase this would be expected to lead to lower intensity of aggregated oviposition, but this is contrary to the observed response to selection. The safest inference, on the basis of the present results, is that neither locomotor activity nor reactivity has an important influence on differences in aggregated oviposition between the selected lines. The high correlation between the number of females observed on individual oviposition sites and the number of eggs laid on those sites, supports the proposition of del Solar \& Ruiz (1992) that environmental heterogeneity alone does not account for the aggregated distribution of eggs. Aggregated oviposition must, at least in part, be due to the contagious distribution of the females themselves.

An implicit assumption made here is that females of the selected strains oviposit at a constant rate. However, D. melanogaster females may engage in bouts of oviposition interspersed by longer intervals when few or no eggs are laid. Eggs are laid in discrete clutches by a number of other species of diptera. Females are capable of retaining fertilized eggs, almost up to the point of hatching, if suitable oviposition sites are unavailable as in crowded culture bottles. Deposition of retained eggs may be rapid once a suitable site has been found, followed by a more constant rate of oviposition later on as females move around. In the experiments reported here, females were regularly transferred to keeping vials containing fresh medium in the period preceding the tests, so that they could lay freely, thus minimizing egg retention. Nevertheless, oviposition in clutches remains as a possible factor contributing to aggregated oviposition. Whether $D$. melanogaster females do oviposit in clutches, and the extent to which physiological as distinct from behavioural differences influence oviposition in the selected populations, requires further investigation.

Points of some interest bearing on the potential impact of female oviposition behaviour on population structure emerge from the relationship between egg density and patch size using grapes as the unit of resource. The indices of dispersion measured for emerging adult progenies show a virtual collapse of the highly significant differences between the $\mathrm{H}$ and $\mathrm{L}$ selected populations evident at oviposition. This is because each grape presents a fixed unit of resource which may be overloaded. If females concentrate their eggs in only a few sites then low survival rates in overcrowded grapes reduce the variance of the distribution of survivors per grape. The effect is greatest in the line selected for a high level of aggregated oviposition. Highly aggregated oviposition would be likely to lead to a highly contagious distribution of adult progenies when the mean level of occupancy per site is low in relation to the carrying capacity of the unit of resource. For $D$. melanogaster at least, patches in the natural environment are likely to vary widely in both size and quality.

Aggregated oviposition behaviour persists even in the population selected for low expression of the trait. It appears to be a norm of the egg-laying behaviour of $D$. melanogaster females, and poses questions regarding its adaptive significance. An interesting indication here may be given by Sang (1949), who showed that larvae exhibit an undercrowding as well as the more familiar overcrowding effect with respect to survival under certain conditions. Larval density can be suboptimal. First-instar larvae feeding as a group in loose association may mutually facilitate the mobilization of resources by physically working the surface of the medium together, or by benefiting from each other's salivary gland secretions onto the substrate. The circumstances under which undercrowding effects occur clearly merit more detailed investigation.

\section{Acknowledgements}

This work was supported by the British Council Academic Links Scheme, and by the University of Sheffield Research Fund, and Fondecyt 1930394 and DID-UACH 5-92-43. The assistance of Sarah L. Johnson and Monica Kibart is gratefully acknowledged.

\section{References}

ATKINSON, w. D. 1983. Gregarious oviposition in Drosophila melanogaster is explained by surface texture. Aust. J. Zool. 36, 925-929.

BRNCIC, D. 1987. Coexistencia de differentes especies de Drosophila en frutas fermentadas naturalmente. Medio Ambiente, 8, 3-9.

BURNET, B., BURNET, L., CONNOLLY, K. AND WILLIAMSON, N. 1988. A genetic analysis of locomotor activity in Drosophila melanogaster. Heredity, 61, 111-119.

CONNOLLY, K. 1968. The social facilitation of preening behaviour in Drosophila melanogaster. Anim. Behav., 16, 385-391. 
DAVID, J. 1970. Oviposition chez Drosophila melanogaster: importance des caractéristiques physiques de la surface de ponte. Rev. Comp. Anim., 4, 70-72.

DAVID, J. R., ALLEMAND, R., HERREWEGE, J. VAN AND COHET, Y. 1983. Ecophysiology: abiotic factors. In: Ashburner, M., Carson, H. L. and Thompson, J. N. (eds) The Genetics and Biology of Drosophila, vol. 3d, pp. 105-170. Academic Press, London.

DEL SOLAR, E. 1968. Selection for and against gregariousness in the choice of oviposition sites by $D$. pseudoobscura. Genetics, 58, 275-282.

DEL SOLAR, E. AND PALOMINo, H. 1966. Choice of oviposition in Drosophila melanogaster. Am. Nat., 100, 127-133.

DEL SOLAR, E. AND RUIZ, G. 1979. Behaviour changes in Drosophila melanogaster in the choice of colored substances for oviposition. Boll. Zool., 46, 17-22.

DEL SOLAR, E. AND RUIZ, G. 1992. Behavioural analysis of the choice of oviposition site by single females of Drosophila melanogaster (Diptera: Drosophilidae). J. Insect Behav., 5, 571-581.

Fogelman, J. C. 1979. Oviposition site preference for substrate temperature in Drosophila melanogaster. Behav. Genet., 9, 407-412.

GELFAND, L. J. AND MCDONALD, J. F. 1980. Relationships between ADH activity and behavioural response to environmental alcohol in Drosophila. Behav. Genet., 10, 237-244.

HOFFMANN, A. A. 1987. A laboratory study of male territoriality in the sibling species Drosophila melanogaster and $D$. simulans. Anim. Behav., 35, 807-818.

JAENIKE, J. 1982. Environmental modification of oviposition behaviour in Drosophila. Am. Nat., 119, 784-802.

KAMBYSELLIS, M. P., STARMER, T., SMATHERS, G. AND HEED, W. B. 1980. Studies of oogenesis in natural populations of Drosophilidae. II. Significance of microclimatic changes on oogenesis of Drosophila mimica. Am. Nat., 115, $67-91$
NAVARRO, J. AND DEL SOLAR, E. 1975. Pattern of spatial distribution in Drosophila melanogaster. Behav. Genet., 5, 9-16.

ROSEWELL, J., SHORROCKS, B. AND EDWARDS, K. 1990. Competition on a divided and ephemeral resource: testing the assumptions. I. Aggregation. J. Anim. Ecol, 59, 9771001.

RUIZ-DUBREUIL, G. AND DEL SOLAR, E. 1986. Effects of selection on oviposition site preference in Drosophila melanogaster. Aust. J. Biol. Sci., 39, 155-160.

RUIZ-DUBREUIL, D. G. AND DEL SOlAR, E. 1991. Genetic influences on gregarious oviposition in Drosophila melanogaster. Evolución Biologica, 5, 161-171.

SANG, J. H. 1949. The ecological determinants of growth in a Drosophila culture. III. Larval and pupal survival. Physiol. Zool., 22, 183-202.

SHORRoCKs, B. 1990. Coexistence in a patchy environment. In: Shorrocks, B. and Swingland, I. R. (eds) Living in a Patchy Environment, pp. 91-106. Oxford University Press, Oxford.

SHORROCKS, B., ROSEWELL, J. AND EDWARDS, K. 1990. Competition on a divided and ephemeral resource: testing the assumptions. II. Association. J. Anim. Ecol, 59, 1003-1017.

SZEBENYI, A. L. 1969. Cleaning behaviour in Drosophila melanogaster. Anim. Behav., 7, 641-651.

TAKAMURA, T. AND FUYAMA, Y. 1980. Behaviour genetics of choice of oviposition sites in Drosophila melanogaster. I. Genetic variability and analysis of behaviour. Behav. Genet., 10, 105-120.

VOLPE, P., CARFAGNA, M. AND DI LORENZO, M. 1967. Extraretinal pigmentation and colour discrimination. I. Choice of colour of substrate in Drosophila melanogaster. J. Exp. Biol., 47, 297-305.

WOGAMAN, D. J. AND SEIGER, M. B. 1983. Light intensity as a factor in the choice of an oviposition site by Drosophila pseudoobscura and Drosophila persimilis. Can. J. Genet. Cytol., 25, 370-377. 